Internat. J. Math. \& Math. Sci.

Vol. 24, No. 10 (2000) 649-661

S0161171200004932

(c) Hindawi Publishing Corp.

\title{
SHALLOW WATER WAVES IN A ROTATING RECTANGULAR BASIN
}

\author{
MOHAMED ATEF HELAL
}

(Received 28 July 1999)

\begin{abstract}
This paper is mainly concerned with the motion of an incompressible fluid in a slowly rotating rectangular basin. The equations of motion of such a problem with its boundary conditions are reduced to a system of nonlinear equations, which is to be solved by applying the shallow water approximation theory. Each unknown of the problem is expanded asymptotically in terms of the small parameter $\epsilon$ which generally depends on some intrinsic quantities of the problem of study. For each order of approximation, the nonlinear system of equations is presented successively. It is worthy to note that such a study has useful applications in the oceanography.
\end{abstract}

Keywords and phrases. Fluid waves, shallow water, rotating fluids.

2000 Mathematics Subject Classification. Primary 76B15, 76U05; Secondary 65N22, 65H10, $65 \mathrm{Y} 10$.

1. Introduction. Rotating fluids is an important topic in both fluid mechanics and oceanography, which attracted numerous scientists and researchers to investigate internal waves, surface waves, tidal waves, and pulses. Oceanographers discovered the importance of coriolis forces and so it must be included in the mathematical model.

Rotating fluids in closed basins simulate many natural free surface flow problems like those of closed seas and lakes. Rotating fluid problems have often been tackled for cylindrical basins using the linear mathematical models (e.g., Brillon and Coulomb [1], Miles [15], Saint-Guily [21]) and rarely by the nonlinear model (cf. Helal and Badawi [9]).

On the contrary, rotating fluids in open seas have merited a lot of attention in the literature since the physical nature of the problem greatly simplifies the solution due to the absence of the side boundaries (cf. LeBlond and Mysak [13], Mei [14]).

To the best of the author's knowledge, the problem of rotating fluids in a parallelepiped basin has been seldom treated, and then only for the linear mathematical model. Consequently, the object of this work is to propose a general framework for the nonlinear analysis of rotating fluids inside a parallelepiped basin, and suggest a mathematical treatment.

However, since the problem is difficult, only the case of shallow water will be considered. Moreover, the powerful perturbation technique will be further adopted. The special case corresponding to the progressive waves will be throughly analyzed.

In the following paragraph a historical background for treatment of rotating fluid problems will be highlighted. A special focus for the pioneering work will be given.

The author believes that the first solutions of the problem of free oscillations in 
rotating rectangular basin of uniform depth were derived by Rayleigh [18, 19]. His study was restricted to slow rotation. Taylor [22] gave the first complete solution for any arbitrary rotation of that rectangular basin. Nevertheless, Lamb [12] gave an approximation for the same problem. The work of Grace [6] represents the first milestone for the rotating rectangular basin where he studied the linear problem and gave solutions for free and forced oscillations, in a rectangular basin. Corkan and Doodson [4] investigated the problem of a square sea by direct numerical integration of the dynamic equations and obtained some interesting results on the so-called symmetric and antisymmetric modes. Moreover, van Dantzig and Lauwerier [23] solved the problem of free oscillation in a rotating rectangular basin for any speed of rotation and they gave also an approximate solution for slow rotation. Rao [17] extended work may be considered to be the second milestone in the analysis of flow in a rotating rectangular basin. Rao showed that the free oscillations consist of symmetric and axisymmetric modes as shown before by Taylor [22]. Moreover, there are modes propagating in the positive and negative direction of the basin. Greenspan [7] gave a detailed and excellent study for the rotating fluids in his famous book. This book is considered as the best reference where the linear and nonlinear problems are given. In his thesis, Suberville [2, 3] presented the linear study for surface and internal waves in rotating circular and rectangular basin. A parallel experimental and theoretical studies were given by Chabert d'Hieres and Suberville [2, 3] for the same problem. In their books, LeBlond and Mysak [13] and Mei [14] have paid considerable attention to rotating fluids and oceans. Pedlosky [16] focused on large-scale flows in the oceans and atmosphere. Renouard, Chabert d'Hieres, and Zhang [20] gave an illustrated experimental study for the strongly nonlinear waves in a rotating system. A theoretical study for the nonlinear gravity waves in a rotating open sea has been presented by Germain and Renouard [5]. Different formulations are discussed for weak, medium, and strong rotations.

It should be underlined however, that most of the above papers were far from the present work, and it is indispensable to give the following work. The present work can be considered as a step for an analytical study for the nonlinear waves in a rotating rectangular basin. Further work including more analytical and numerical study will be reported in a forthcoming work.

2. The problem. Consider an incompressible perfect fluid in a rectangular basin. The domain occupied by the fluid is defined as:

$$
0 \leq z \leq \zeta, \quad-a \leq x \leq a, \quad-b \leq y \leq b .
$$

The basin rotates around its $z$-axis with angular velocity $\Omega_{c}$.

The present work concentrates on providing the governing equations for the free surface. Throughout this paper the following notation represent the given definitions (cf. Figure 2.1).

$M(x, y, z)$ : the rectangular coordinates of the point $M$

$H$ : thickness at rest of the fluid

$\rho$ : constant density of the fluid 


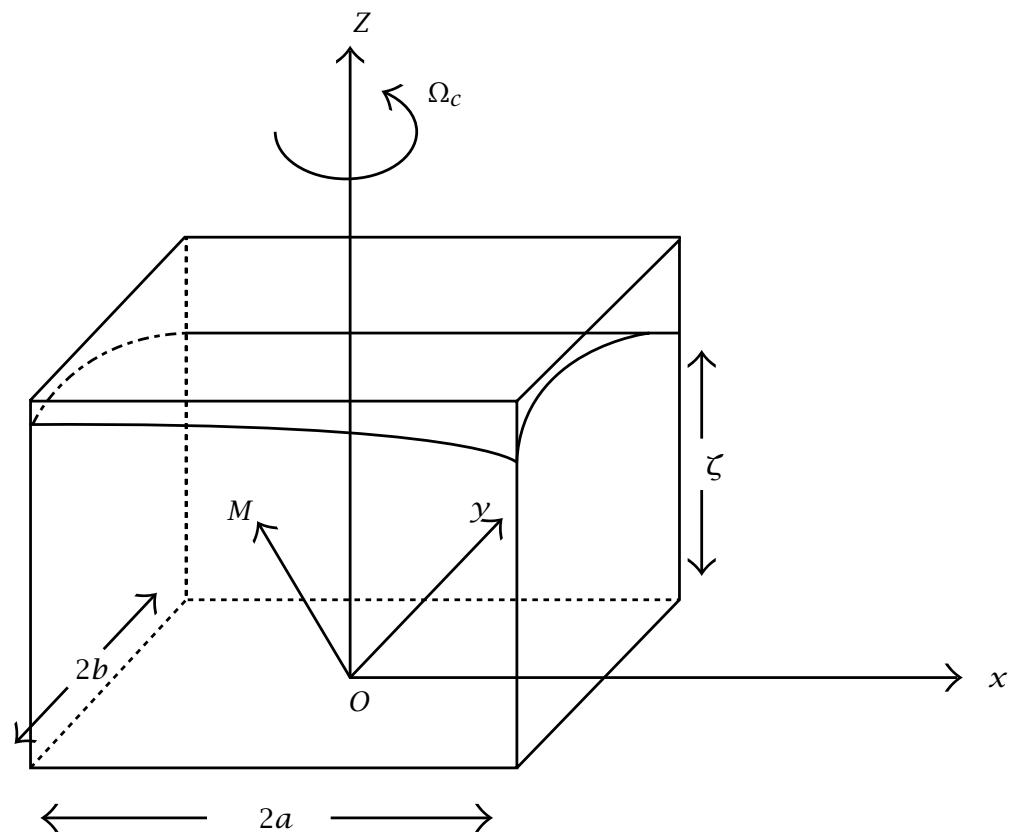

FIGURE 2.1.

$p$ : pressure of the fluid

$\mathbf{V}=\left(V_{x}, V_{y}, V_{z}\right)$ : the velocity vector

$z=\zeta(x, y, t)$ : free surface equation

$\Omega_{c}$ : the angular velocity of the system

$g$ : the acceleration of gravity.

\section{Basic equations}

3.1. Hydrodynamic equations. As we are dealing with a rectangular basin, the equations can be expressed in vector form as follows (using rectangular coordinates):

The dynamic equations

$$
\rho\left[\frac{\partial \mathbf{V}}{\partial t}+(\mathbf{V} \cdot \nabla) \mathbf{V}+2 \Omega_{c} \wedge \mathbf{V}+\Omega_{c} \wedge\left(\Omega_{c} \wedge \overrightarrow{O M}\right)+\nabla g z\right]=-\nabla p
$$

The continuity equation

$$
\operatorname{div} \mathbf{V}=0
$$

where

$$
\mathbf{V}=V_{x} \mathbf{i}+V_{y} \mathbf{j}+V_{z} \mathbf{k}, \quad \overrightarrow{O M}=x \mathbf{i}+y \mathbf{j}+z \mathbf{k}, \quad \Omega_{c}=\Omega_{c} \mathbf{k}
$$

and $\{\mathbf{i}, \mathbf{j}, \mathbf{k}\}$ are the unit vectors in a rectangular coordinate system. 
Equations (3.1) and (3.2) can be rewritten in their scalar form as:

$$
\begin{gathered}
\frac{\partial V_{x}}{\partial x}+\frac{\partial V_{y}}{\partial y}+\frac{\partial V_{z}}{\partial z}=0 \\
\frac{\partial V_{x}}{\partial t}+\left(V_{x} \frac{\partial V_{x}}{\partial x}+V_{y} \frac{\partial V_{x}}{\partial y}+V_{z} \frac{\partial V_{x}}{\partial z}\right)-2 \Omega_{c} V_{y}-2 x \Omega_{c}^{2}=-\frac{1}{\rho} \frac{\partial p}{\partial x} \\
\frac{\partial V_{y}}{\partial t}+\left(V_{x} \frac{\partial V_{y}}{\partial x}+V_{y} \frac{\partial V_{y}}{\partial y}+V_{z} \frac{\partial V_{y}}{\partial z}\right)+2 \Omega_{c} V_{x}-2 y \Omega_{c}^{2}=-\frac{1}{\rho} \frac{\partial p}{\partial y} \\
\frac{\partial V_{z}}{\partial t}+\left(V_{x} \frac{\partial V_{z}}{\partial x}+V_{y} \frac{\partial V_{z}}{\partial y}+V_{z} \frac{\partial V_{z}}{\partial z}\right)+g=-\frac{1}{\rho} \frac{\partial p}{\partial z}
\end{gathered}
$$

3.2. Boundary conditions. We assume that all the boundaries of the domain are impermeable, including the free surface which is unknown.

(i) At the bottom:

$$
V_{z}=0 \text { for } z=0
$$

(ii) On the vertical boundaries of the basin:

$$
V_{x}=0 \quad \text { for } x= \pm a, \quad V_{y}=0 \quad \text { for } y= \pm b .
$$

(iii) On the free surface $z=\zeta(x, y, t)$ we have two conditions. The first describes the impermeability

$$
\left[V_{z}-\frac{\partial \zeta}{\partial t}-V_{x} \frac{\partial \zeta}{\partial x}-V_{y} \frac{\partial \zeta}{\partial y}\right]_{z=\zeta}=0
$$

The second condition describes its isoparity

$$
p(x, y, z=\zeta, t)=p_{\mathrm{at}}=\text { constant }
$$

where $p_{\text {at }}$ represents the atmospheric pressure which can be taken as zero without any loss of generality.

Equations (3.4), (3.5), (3.6), (3.7), and (3.8) describe completely the governing equations for the nonlinear problem.

4. The shallow water theory. From now on, we consider the case of rectangular basin with very large horizontal dimensions ( $x$ and $y$ ) compared with its vertical height $(z)$. Applying the shallow water approximation theory (see Helal [8] and Helal and Badawi [9]), which designates the following new distorted parameters:

$$
\bar{x}=\epsilon \mathcal{X}, \quad \bar{y}=\epsilon y, \quad \bar{z}=z, \quad \tau=\epsilon t,
$$


where $\epsilon$ is a small parameter which can depend on the ratio between the vertical height and $(a+b) / 2$. Moreover, we are interested in the case of slow rotation, that is,

$$
\Omega_{c}=\epsilon \Omega
$$

For simplicity, hereafter, we use $u, v, w$ instead of $V_{x}, V_{y}, V_{z}$.

The system of equations (3.4), (3.5), (3.6), (3.7), and (3.8) (after using the distorted parameters) can be written as

$$
\begin{gathered}
\epsilon\left(\frac{\partial u}{\partial \tau}+u \frac{\partial u}{\partial \bar{x}}+v \frac{\partial u}{\partial \bar{y}}-2 \Omega v-\bar{x} \Omega^{2}\right)+w \frac{\partial u}{\partial \bar{z}}=-\frac{\epsilon}{\rho} \frac{\partial p}{\partial \bar{x}} \\
\epsilon\left(\frac{\partial v}{\partial \tau}+u \frac{\partial v}{\partial \bar{x}}+v \frac{\partial v}{\partial \bar{y}}+2 \Omega u-\bar{y} \Omega^{2}\right)+w \frac{\partial v}{\partial \bar{z}}=-\frac{\epsilon}{\rho} \frac{\partial p}{\partial \bar{y}}, \\
\epsilon\left(\frac{\partial w}{\partial \tau}+u \frac{\partial w}{\partial \bar{x}}+v \frac{\partial w}{\partial \bar{y}}\right)+w \frac{\partial w}{\partial z}+g=-\frac{1}{\rho} \frac{\partial p}{\partial \bar{z}} \\
\epsilon\left(\frac{\partial u}{\partial \bar{x}}+\frac{\partial v}{\partial \bar{y}}\right)+\frac{\partial w}{\partial \bar{z}}=0, \\
(w)_{\bar{z}=0}=0, \\
(u)_{\bar{x}= \pm A}=0, \quad(v)_{\bar{y}= \pm B}=0, \quad \text { where } A=\epsilon a, B=\epsilon b \\
{[p(\bar{x}, \bar{y}, \bar{z}, \tau)]_{\bar{z}=\zeta}=0,} \\
{\left[w-\epsilon\left(\frac{\partial \zeta}{\partial \tau}+u \frac{\partial \zeta}{\partial \bar{x}}+v \frac{\partial \zeta}{\partial \bar{y}}\right)\right]_{\bar{z}=\zeta}=0 .}
\end{gathered}
$$

Expanding the dependent variables in the neighborhood of the undisturbed uniform state in general asymptotic series, will lead to two sets of identical equations, and due to the parity of the system of equations, we take the more suitable expansions for the dependent variables in the form (cf. Helal and Badawi [9]):

$$
\begin{aligned}
V_{x} & =u=\sum_{n=1}^{\infty} \epsilon^{2 n} u_{2 n}(\bar{x}, \bar{y}, \bar{z}, \tau), \\
V_{y} & =v=\sum_{n=1}^{\infty} \epsilon^{2 n} v_{2 n}(\bar{x}, \bar{y}, \bar{z}, \tau), \\
V_{z} & =w=\sum_{n=0}^{\infty} \epsilon^{2 n+1} w_{2 n+1}(\bar{x}, \bar{y}, \bar{z}, \tau), \\
p & =\sum_{n=0}^{\infty} \epsilon^{2 n} p_{2 n}(\bar{x}, \bar{y}, \bar{z}, \tau), \\
\zeta & =\sum_{n=0}^{\infty} \epsilon^{2 n} \zeta_{2 n}(\bar{x}, \bar{y}, \tau),
\end{aligned}
$$

where the values of $\zeta_{0}$ and $p_{0}$ have the forms (from studying the stationary state [9]):

$$
\zeta_{0}=H+\frac{\Omega_{c}^{2}}{4 g}\left(x^{2}+y^{2}\right), \quad p_{0}=-\rho g(\bar{z}-\zeta) .
$$


For simplicity, we omit the bar sign throughout the rest of the paper.

The boundary conditions on the free surface and on the interface can be expanded in the form:

$$
[F]_{z=z_{0}+\epsilon D}=[F]_{z_{0}}+\epsilon D\left[\frac{\partial F}{\partial z}\right]_{z=z_{0}}+\frac{(\epsilon D)^{2}}{2 !}\left[\frac{\partial^{2} F}{\partial z^{2}}\right]_{z=z_{0}}+\cdots
$$

Expanding all the boundary conditions in a similar manner to that of (4.6) and making use of (4.4) and (4.5) in the system of (3.4), (3.5), (3.6), (3.7), and (3.8); this will lead to an ordered set of equations to be solved in the sequel.

\section{Orders of approximations}

5.1. First order approximation. The equation

$$
\frac{\partial w_{1}}{\partial z}=0 \quad \text { with the boundary condition }\left[w_{1}\right]_{z=0}=0
$$

leads to

$$
w_{1}=0
$$

5.2. Second order approximation. For this approximation, we have

$$
-\frac{1}{\rho} \frac{\partial p_{2}}{\partial z}=0
$$

and from the conditions of isoparity on free surface we get

$$
\left[p_{2}-\rho g \zeta_{2}\right]_{z=\zeta_{0}}=0
$$

which implies that

$$
p_{2}=\rho g \zeta_{2}
$$

5.3. Third order approximation. This approximation gives

$$
\begin{aligned}
& \frac{\partial u_{2}}{\partial \tau}-2 \Omega v_{2}=-\frac{1}{\rho} \frac{\partial p_{2}}{\partial x}, \\
& \frac{\partial v_{2}}{\partial \tau}+2 \Omega u_{2}=-\frac{1}{\rho} \frac{\partial p_{2}}{\partial y}, \\
& \frac{\partial u_{2}}{\partial x}+\frac{\partial v_{2}}{\partial y}+\frac{\partial w_{3}}{\partial z}=0
\end{aligned}
$$

with the following boundary conditions:

$$
\left[w_{3}\right]_{z=0}=0, \quad\left[w_{3}-\left(\frac{\partial \zeta_{2}}{\partial \tau}+u_{2} \frac{\partial \zeta_{0}}{\partial x}+v_{2} \frac{\partial \zeta_{0}}{\partial y}\right)\right]_{z=\zeta_{0}}=0
$$


After some calculations, we can deduce that $u_{2}$ and $v_{2}$ are functions of $x, y$, and $\tau$ only.

From (5.8) and the boundary conditions (5.9), we get

$$
w_{3}=-z\left(\frac{\partial u_{2}}{\partial x}+\frac{\partial v_{2}}{\partial y}\right)
$$

After extensive manipulation, we get the following partial differential equation for $p_{2}$ :

$$
\begin{aligned}
& {\left[\frac{\Omega^{2}}{2}\left(x^{2}+y^{2}\right)+H g\right] \frac{\partial}{\partial \tau} \nabla^{2} p_{2}} \\
& \quad+\Omega^{2}\left[\frac{\partial}{\partial \tau}\left(x \frac{\partial p_{2}}{\partial x}+y \frac{\partial p_{2}}{\partial y}\right)+2 \Omega\left(x \frac{\partial p_{2}}{\partial y}-y \frac{\partial p_{2}}{\partial x}\right)\right]=\left(\frac{\partial^{3}}{\partial \tau^{3}}+4 \Omega^{2} \frac{\partial}{\partial \tau}\right) p_{2}
\end{aligned}
$$

The following system of equations gives the relation between $p_{2}$ and $u_{2}$ and $v_{2}$ :

$$
\begin{aligned}
& \left(4 \Omega^{2}+\frac{\partial^{2}}{\partial \tau^{2}}\right) u_{2}=-\frac{1}{\rho}\left(\frac{\partial^{2} p_{2}}{\partial x \partial \tau}+2 \Omega \frac{\partial p_{2}}{\partial y}\right) \\
& \left(4 \Omega^{2}+\frac{\partial^{2}}{\partial \tau^{2}}\right) v_{2}=-\frac{1}{\rho}\left(\frac{\partial^{2} p_{2}}{\partial y \partial \tau}-2 \Omega \frac{\partial p_{2}}{\partial x}\right)
\end{aligned}
$$

Solving the partial differential equation (5.11) gives $p_{2}$ and consequently by using the last system of (5.12) we can deduce $u_{2}$ and $v_{2}$. We must notice that the solution of the partial differential equation (5.11) governing the unknown $p_{2}$ is very complicated and needs further research.

6. Progressive waves. In this section, we pay attention to the study of progressive waves (see Helal and Badawi [9]). It has been shown that all unknowns $p_{2}, u_{2}$, and $v_{2}$ are functions of $x, y$, and $\tau$ only. In the present case, we introduce the parameter $\Theta=\theta(x, y)-\omega \tau$, where $\omega$ is the pulse angular frequency.

The unknowns $p_{2}$ can be expanded as a Fourier series in $\Theta$ as follows:

$$
p_{2}=\sum_{n=0}^{\infty}\left[a_{n}(x, y) \cos n \Theta+b_{n}(x, y) \sin n \Theta\right] .
$$

After substitution of $p_{2}$ into the system of equations (5.12), and manipulation, we obtain two partial differential equations for $u_{2}$ and $v_{2}$; the general solution of which are given by

$$
\begin{aligned}
& u_{2}=\sum_{n=0}^{\infty}\left[\alpha_{n}(x, y) \cos n \Theta+\beta_{n}(x, y) \sin n \Theta\right], \\
& v_{2}=\sum_{n=0}^{\infty}\left[\gamma_{n}(x, y) \cos n \Theta+\delta_{n}(x, y) \sin n \Theta\right],
\end{aligned}
$$


where

$$
\begin{aligned}
& \alpha_{n}=-\frac{1}{\rho\left(4 \Omega^{2}-n^{2} \omega^{2}\right)}\left(-\omega n \frac{\partial b_{n}}{\partial x}+2 \Omega \frac{\partial a_{n}}{\partial y}+\omega n^{2} a_{n} \frac{\partial \Theta}{\partial x}+2 \Omega n b_{n} \frac{\partial \Theta}{\partial y}\right), \\
& \beta_{n}=-\frac{1}{\rho\left(4 \Omega^{2}-n^{2} \omega^{2}\right)}\left(\omega n \frac{\partial a_{n}}{\partial x}+2 \Omega \frac{\partial b_{n}}{\partial y}+\omega n^{2} b_{n} \frac{\partial \Theta}{\partial x}-2 \Omega n a_{n} \frac{\partial \Theta}{\partial y}\right), \\
& \gamma_{n}=-\frac{1}{\rho\left(4 \Omega^{2}-n^{2} \omega^{2}\right)}\left(-\omega n \frac{\partial b_{n}}{\partial y}-2 \Omega \frac{\partial a_{n}}{\partial x}+\omega n^{2} a_{n} \frac{\partial \Theta}{\partial y}-2 \Omega n b_{n} \frac{\partial \Theta}{\partial x}\right), \\
& \delta_{n}=-\frac{1}{\rho\left(4 \Omega^{2}-n^{2} \omega^{2}\right)}\left(\omega n \frac{\partial a_{n}}{\partial y}-2 \Omega \frac{\partial b_{n}}{\partial x}+\omega n^{2} b_{n} \frac{\partial \Theta}{\partial y}+2 \Omega n a_{n} \frac{\partial \Theta}{\partial x}\right),
\end{aligned}
$$

where

$$
\frac{\partial \Theta}{\partial x}=-\frac{y}{x^{2}+y^{2}}, \quad \frac{\partial \Theta}{\partial y}=\frac{x}{x^{2}+y^{2}}
$$

It is to be noted that due to the periodicity of solutions of (5.6) and (5.7), the quantity $\left(4 \Omega^{2}-n^{2} \omega^{2}\right)$ never vanishes. If we substitute from (6.1) into (5.11) we get the following ordinary differential equation which determines the unknown coefficients $a_{n}(x, y)$ and $b_{n}(x, y)$ :

$$
\begin{aligned}
&\left(\frac{\Omega^{2}}{2}\left(x^{2}+y^{2}\right)+H g\right)\left(n \omega \nabla^{2} a_{n}+\frac{n^{3} \omega}{x^{2}+y^{2}} a_{n}\right)+\Omega^{2} n \omega\left(x \frac{\partial a_{n}}{\partial x}+y \frac{\partial a_{n}}{\partial y}\right)-2 n \Omega^{3} a_{n}-\left((-n \omega)^{3}+4 \Omega^{2} n \omega\right) a_{n} \\
&=-\left(\frac{\Omega^{2}}{2}\left(x^{2}+y^{2}\right)+H g\right) \frac{2 n^{2} \omega\left(-y\left(\partial b_{n} / \partial x\right)+x\left(\partial b_{n} / \partial y\right)\right)}{x^{2}+y^{2}} \\
&- 2 \Omega^{3}\left(x \frac{\partial b_{n}}{\partial y}-y \frac{\partial b_{n}}{\partial x}\right), \\
&\left(\frac{\Omega^{2}}{2}\left(x^{2}+y^{2}\right)+H g\right)\left(-n \omega \nabla^{2} b_{n}+\frac{n^{3} \omega}{x^{2}+y^{2}} b_{n}\right)-\Omega^{2} n \omega\left(x \frac{\partial b_{n}}{\partial x}+y \frac{\partial b_{n}}{\partial y}\right) \\
&+2 n \Omega^{3} b_{n}+\left[(-n \omega)^{3}+4 \Omega^{2} n \omega\right] b_{n} \\
&=-\left(\frac{\Omega^{2}}{2}\left(x^{2}+y^{2}\right)+H g\right) \frac{2 n^{2} \omega\left(-y\left(\partial a_{n} / \partial x\right)+x\left(\partial a_{n} / \partial y\right)\right)}{x^{2}+y^{2}} \\
&-2 \Omega^{3}\left(x \frac{\partial a_{n}}{\partial y}-y \frac{\partial a_{n}}{\partial x}\right) .
\end{aligned}
$$

Also, since the basin's vertical walls, $x= \pm a$ and $y= \pm b$, are impermeable so the functions $u_{2}$ and $v_{2}$ must vanish at these walls, that is,

$$
\left[u_{2}\right]_{x= \pm a}=0, \quad\left[v_{2}\right]_{y= \pm b}=0
$$


which gives:

$$
\begin{aligned}
& {\left[-\omega n \frac{\partial b_{n}}{\partial x}+2 \Omega \frac{\partial a_{n}}{\partial y}-a_{n} \omega n^{2}\left(\frac{y}{x^{2}+y^{2}}\right)+2 \Omega n b_{n}\left(\frac{x}{x^{2}+y^{2}}\right)\right]_{x= \pm a}=0} \\
& {\left[\omega n \frac{\partial a_{n}}{\partial x}+2 \Omega \frac{\partial b_{n}}{\partial y}-b_{n} \omega n^{2}\left(\frac{y}{x^{2}+y^{2}}\right)-2 \Omega n a_{n}\left(\frac{x}{x^{2}+y^{2}}\right)\right]_{x= \pm a}=0} \\
& {\left[-\omega n \frac{\partial b_{n}}{\partial y}-2 \Omega \frac{\partial a_{n}}{\partial x}+a_{n} \omega n^{2}\left(\frac{x}{x^{2}+y^{2}}\right)+2 \Omega n b_{n}\left(\frac{y}{x^{2}+y^{2}}\right)\right]_{y= \pm b}=0} \\
& {\left[\omega n \frac{\partial a_{n}}{\partial y}-2 \Omega \frac{\partial b_{n}}{\partial x}+b_{n} \omega n^{2}\left(\frac{x}{x^{2}+y^{2}}\right)-2 \Omega n a_{n}\left(\frac{y}{x^{2}+y^{2}}\right)\right]_{y= \pm b}=0 .}
\end{aligned}
$$

Since the solution must be bounded at the origin, $a_{n}(0)$ and $b_{n}(0)$ should be bounded. Now we are left with a system of partial differential equations with variable coefficients, namely, equations (6.5), to be solved. Our purpose is to obtain a bounded solution for this system of partial differential equations (6.5) which satisfy the boundary conditions (6.7). The solution gives the unknowns $a_{n}$ and $b_{n}$ which represent the Fourier coefficients of the function $p_{2}$ and consequently we can deduce the other unknowns $u_{2}$ and $v_{2}$. This completes the solution at this stage of approximation. However, the analytical solution of the system of partial differential equation is very complicated, so we think that the numerical solution of this system of partial differential equations with the boundary condition will be necessary.

7. Algorithm for the numerical solution of the above system. The coupled system of partial differential equations (6.5) for the unknowns $a_{n}(x, y)$ and $b_{n}(x, y)$ and conditions (6.7) can be approximately replaced by a corresponding system of algebraic equations using the following procedure:

(1) divide the finite interval $(-a<x<a)$ into a finite number $N$ of equal subintervals $\triangle x$ with length $2 a / N$,

(2) divide the finite interval $(-b<y<b)$ into a finite number $M$ of equal subintervals $\triangle y$ with length $2 b / M$,

(3) replace each partial derivative of the unknown functions $a_{n}(x, y)$ and $b_{n}(x, y)$ by a suitable finite differences (see Isaacson and Keller [10]), thus leading to a system of algebraic equations,

(4) insert the discretised boundary conditions into the algebraic system of equations,

(5) use any appropriate technique to get the numerical solution of the resulting linear algebraic system of equations.

8. Numerical applications. In this section, we give a numerical solution to the above problem, for some particular case of a square basin $(a=b)$. We use the following nondimensional parameters: 
$\tilde{\Omega}=\frac{\Omega}{\Omega_{0}}, \quad \tilde{w}=\frac{w}{\Omega_{0}}, \quad \tilde{a}=\frac{a \Omega_{0}}{\sqrt{g H}}, \quad \tilde{x}=\frac{x \Omega_{0}}{\sqrt{g H}}, \quad \tilde{y}=\frac{y \Omega_{0}}{\sqrt{g H}}, \quad \tilde{\rho}=\frac{\rho}{\rho_{0}}, \quad \tilde{\tau}=\Omega_{0} \tau$,

where $\Omega_{0}, \rho_{0}$, and $H$ are the characteristic angular velocity, density, and height, respectively.

In our application, we considered the principal harmonics $(n=1)$.

The numerical solution is obtained for the following two sets of numerical data:

(1) $\tilde{\Omega}=1, \tilde{a}=1, \tilde{\rho}=1$, and $\tilde{w}=0.5$,

(2) $\tilde{\Omega}=1, \tilde{a}=1, \tilde{\rho}=1$, and $\tilde{w}=-0.5$.

The first set of data corresponds to the $P$ mode (which describe waves traveling with the flow) (cf. Yih [24]), while the second one represents the $N$ mode (which describe waves traveling against the flow).

Here, Figures 8.1a, 8.1b, 8.1c, 8.1d illustrate the pattern of $\zeta_{2}$ for the $P$ mode at different instants of time, whilst Figures $8.2 \mathrm{a}, 8.2 \mathrm{~b}, 8.2 \mathrm{c}, 8.2 \mathrm{~d}$ give the $N$ mode at the same different instants of time.

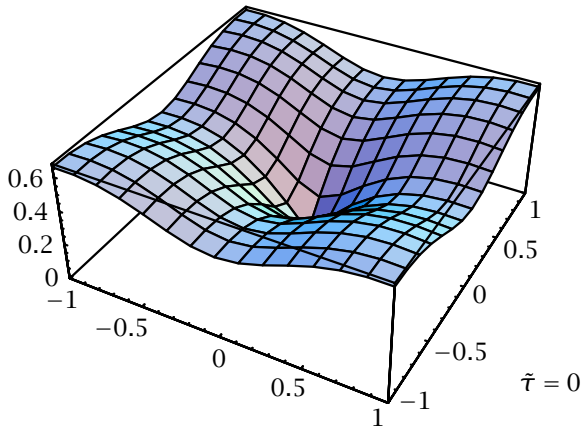

(a)

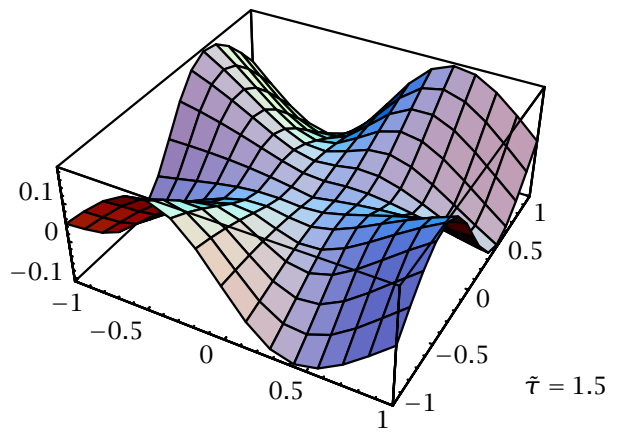

(c)

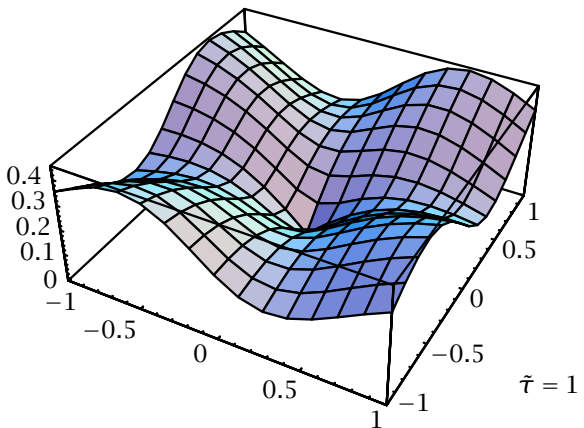

(b)

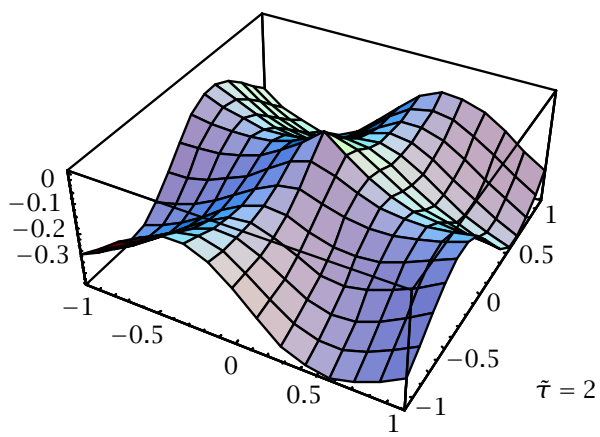

(d)

FIGURE 8.1. 


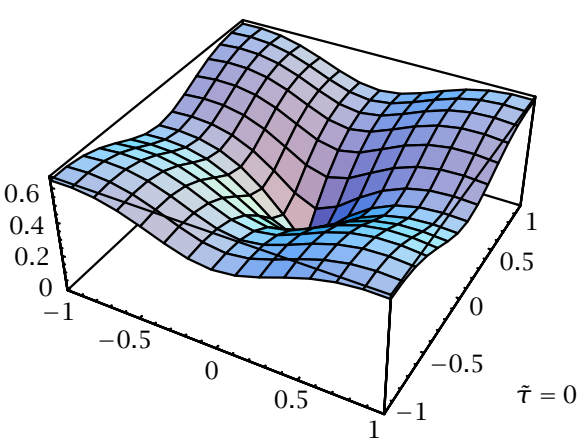

(a)

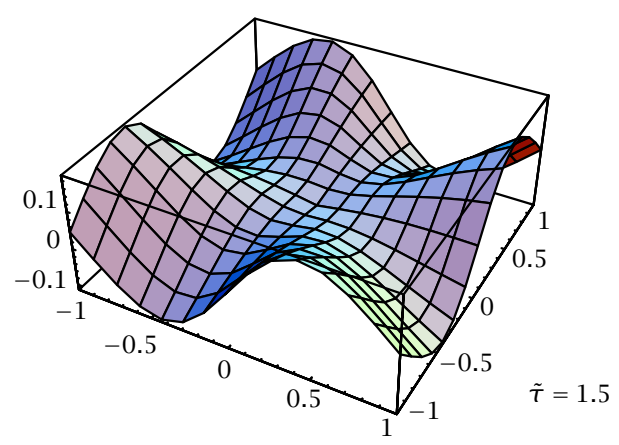

(c)

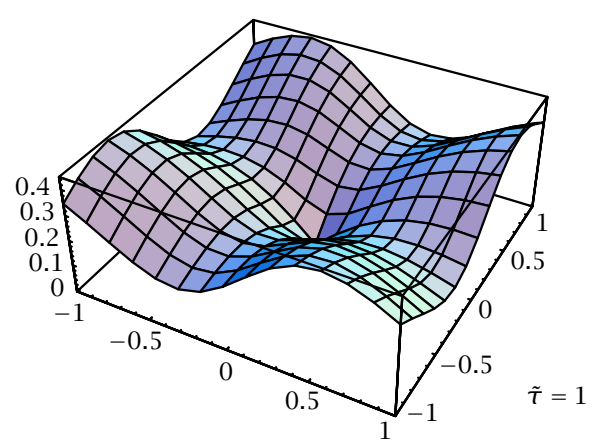

(b)

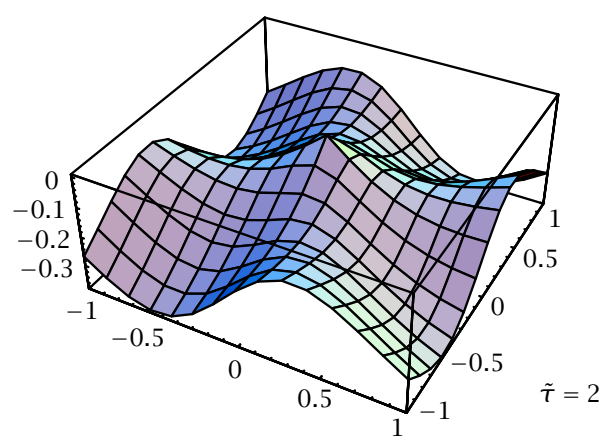

(d)

FIGURE 8.2.

9. Discussions and conclusions. Numerous works are available which handle the application of linear shallow water waves in the oceanography and fluid mechanics topics. It is well known that there are two types of waves, one is a family of the so-called Poincaré waves and the other is the Kelvin waves (cf. Pedlosky [16] and Kundu [11]). The free surface attains a parabolic shape due to the balance between the centrifugal force and the gravity force. However, the nonlinear treatment of these problems is seldom found. For this reason, the present work introduces a mathematical model for the nonlinear shallow water equations. It is difficult to obtain an analytic solution for such problems, while the numerical treatment is possible. Although this problem for the square basin seems to have many difficulties, it may be more suitable for oceanographical applications.

The mathematical model handles ideal fluid as a reasonable approximation for the actual fluid in physical oceanography. It is worthy to search for a simple physical oceanography and to formulate the corresponding mathematical model. The closed seas and lakes distributed all over the world represent good examples for applications. These include the Caspian and Aral seas in Asia, Winnipeg and Ontario in North 
America, Lago de Nicaragua in South America, as well as the lakes of Chad and Victoria in Africa.

Thus it can be concluded that the proposed mathematical model can be employed for handling such real lakes and/or closed seas. As a first approximation, each of these cases can be approximated by a rectangular form having its real dimensions and locations. Next, a numerical solution for the proposed mathematical model could be attained as explained before. Moreover, these results can be further compared to other computational models and/or experimental data. This comparison resembles an important verification and testimony to this mathematical model.

\section{REFERENCES}

[1] M. Brillouin and J. Coulomb, Oscillation d'un Liquide Pesant dans un Bassin Cylinderique en Rotation, Gautier-Villars, Paris, 1933.

[2] G. Chabert d'Hieres and J. L. Suberville, A theoretical and experimental study of internal waves in a rotating stratified medium, Theoret. Appl. Mech., Proc. 14th IUTAM Congr. (1976), 393-405. Zbl 369.76024.

[3] __ Etude theorique et experimentale des ondes internes en milieu stratifie tournant, Oceanologie 9 (1978), 53-72.

[4] R. H. Corkan and A. T. Doodson, Free tidal oscillations in a rotating square sea, Proc. Roy. Soc. London Ser. A 215 (1952), 147-162. MR 16,972j. Zbl 048.19605.

[5] J. P. Germain and D. P. Renouard, On permanent nonlinear waves in a rotating fluids, Fluid Dynam. Res. 7 (1991), 263-278.

[6] S. F. Grace, Tidal oscillation in rotating rectangular basins of uniform depth, Monthly Notices Roy. Astronom. Soc. 2 (1931), no. 8, 386-398, Geophysical Supplement.

[7] H. P. Greenspan, The Theory of Rotating Fluids, Cambridge Monographs on Mechanics and Applied Mathematics, Cambridge University Press, Cambridge, 1968. Zbl 182.28103 .

[8] M. A. Helal, Application de la theorie de l'eau peu profond au cas des fluides en bicouche et des fluides en milieux tournants, Ph.D. thesis, Cairo University, Faculty of Science, Giza, Egypt, 1981.

[9] M. A. Helal and S. Badawi, Waves in a rotating stratified shallow water, Nuovo Cimento B 104 (1989), no. 3, 245-262.

[10] E. Isaacson and H. B. Keller, Analysis of Numerical Methods, John Wiley \& Sons Inc., New York, London, Sydney, 1966. MR 34\#924. Zbl 168.13101.

[11] P. K. Kundu, Fluid Mechanics, Academic Press, Inc., San Diego, 1990. Zbl 780.76001.

[12] Sir H. Lamb, Hydrodynamics, 5th ed., Cambridge University Press, Cambridge, 1924.

[13] P. H. LeBlond and L. A. Mysak, Waves in the Oceans, Elsevier, Amsterdam, 1978.

[14] C. C. Mei, The Applied Dynamics of Ocean Surface Waves, John Wiley \& Sons, New York, N.Y., 1983.

[15] J. W. Miles, Free surface oscillations in a rotating liquid, Phys. Fluids 2 (1959), 297-305. MR 22\#7484. Zbl 085.41002.

[16] J. Pedlosky, Geophysical Fluid Dynamics, 2nd ed., Springer-Verlag, Berlin, New York, 1987. Zbl 713.76005 .

[17] R. Rao, Free gravitational oscillations in rotating rectangular basin, J. Fluid Mech. 25 (1966), no. 3, 523-555.

[18] L. Rayleigh, On the vibrations of rectangular sheet of rotating liquid, Phil. Mag. 5 (1903), 297-301.

[19]_ Notes concerning tidal oscillation upon a rotating globe, Proc. Roy. Soc. London Ser. A 82 (1909), 448-464.

[20] D. Renouard, G. Chabert d'Hieres, and X. Zhang, An experemental study of strongly nonlinear waves in a rotating system, J. Fluid Mech. 177 (1987), 381-394. 
[21] B. Saint-Guily, La theorie des ondes liquides de gravite dans un bassin tournant, La Houille Blanche 5 (1963), 629-634.

[22] G. I. Taylor, Tidal oscillations in gulfs and rectangular basins, Proc. London Math. Soc. 20 (1922), 148-181.

[23] D. van Dantzig and H. A. Lauwerier, The North Sea problem. IV. Free oscillations of a rotating rectangular sea, Nederl. Akad. Wetensch. Proc. Ser. A 6322 (1960), 339354. MR 23\#B2031. Zbl 097.41903.

[24] C. S. Yih, Solitary waves in Poiseuille flow of a rotating fluid, Quart. Appl. Math. 52 (1994), no. 4, 739-752. MR 95i:76108. Zbl 836.76096.

Mohamed Atef Helal: Department of Mathematics, Faculty of Science, Cairo UniverSITY, GIZA, EGYPT

E-mail address: mahe1a1@frcu.eun.eg 


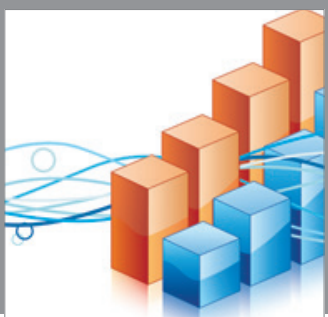

Advances in

Operations Research

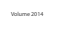

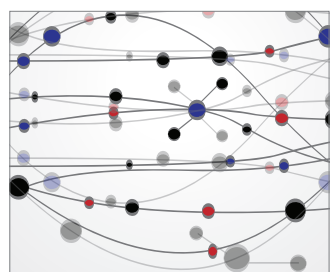

\section{The Scientific} World Journal
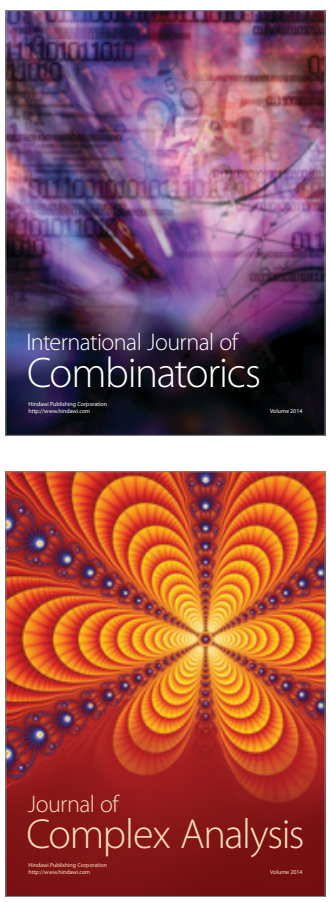

International Journal of

Mathematics and

Mathematical

Sciences
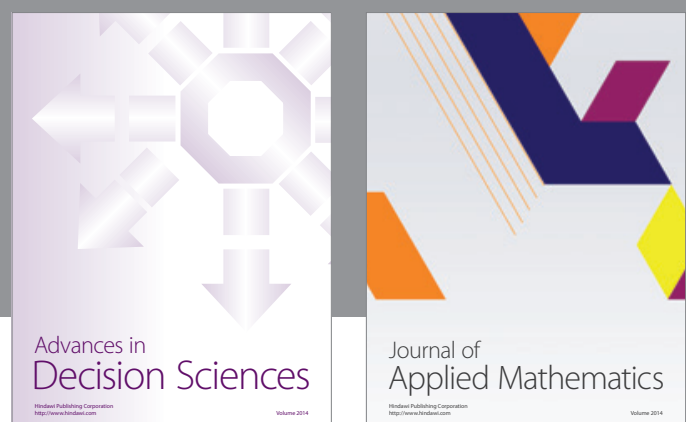

Journal of

Applied Mathematics
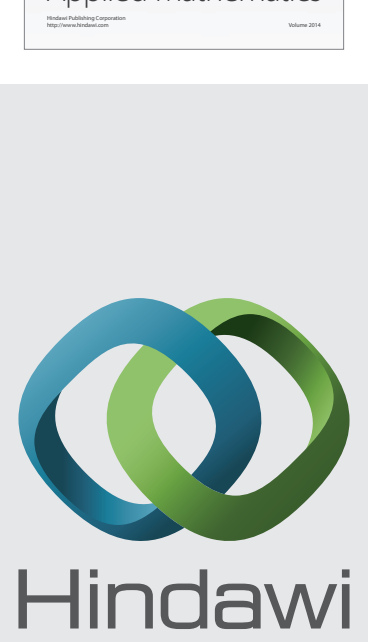

Submit your manuscripts at http://www.hindawi.com
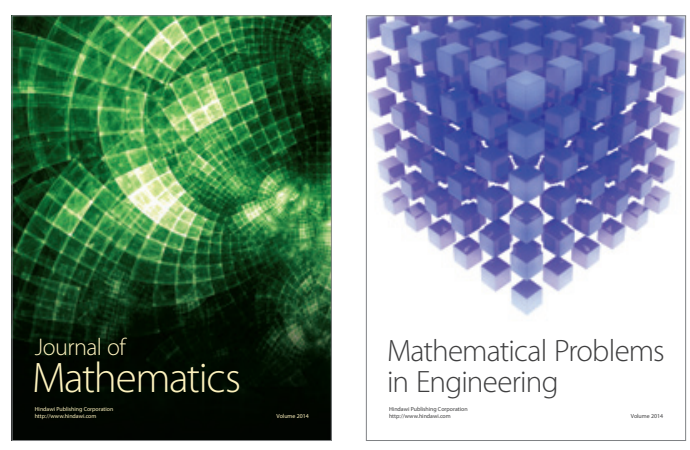

Mathematical Problems in Engineering
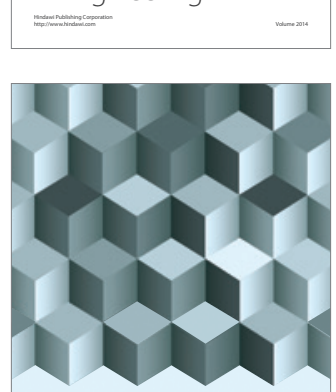

Journal of

Function Spaces
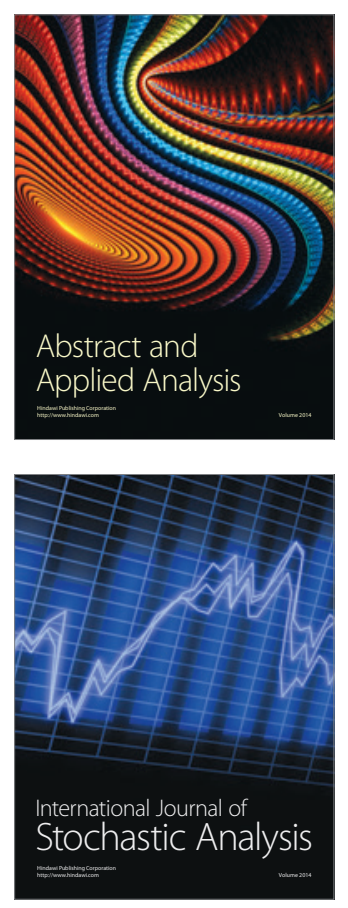

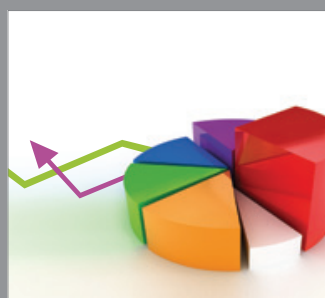

ournal of

Probability and Statistics

Promensencen
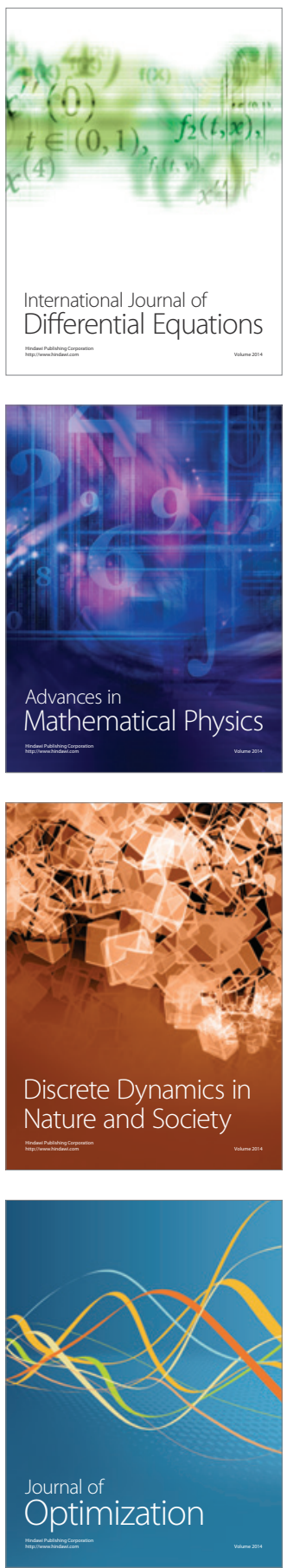